\title{
INTERVENÇÕES EM PSICODRAMA: UMA REVISÃO SISTEMÁTICA
}

\author{
Vandro Antonio Fernandes ${ }^{1, *}$ (D), Cláudia Mara Bosetto Cenci' (D), \\ Icaro Bonamigo Gaspodini² (D)
}

\section{RESUMO}

O Psicodrama busca conhecer a realidade apresentada, investigando a psique humana através da dramatização. Este estudo objetiva identificar intervenções em psicodrama, suas limitações e resultados. Nesta revisão sistemática da literatura, buscaram-se trabalhos publicados entre 2009 e 2019, no Portal de Periódicos da Capes. Dos 1403 estudos encontrados, 34 atenderam aos critérios de inclusão e exclusão. Após a análise dos artigos emergiram 11 categorias e eles foram agrupados por similaridade de assuntos ou público participante. O psicodrama se mostrou eficiente na maioria das intervenções, salientando sua flexibilidade e abrangência, permitindo mudanças significativas na vida dos participantes, reflexões sobre padrões comportamentais e autoconhecimento. As limitações encontradas ressaltam escassez de recursos, aparentemente financeiros, como questão comum a inúmeros artigos.

PALAVRAS-CHAVE: Psicodrama; Sociodrama; Intervenções; Limitações; Resultados.

\section{PSYCHODRAMA INTERVENTIONS: A SYSTEMATIC REVIEW}

\begin{abstract}
Psychodrama seeks to know the reality presented, investigating the human psyche through dramatization. This study aims to identify interventions in psychodrama, its limitations and results. This systematic review of the literature sought works published between 2009 and 2019, on the Portal de Periódicos da Capes. In 1403 studies found, 34 met the inclusion and exclusion criteria. After analyzing the articles, 11 categories emerged and they were grouped by similarity of subjects or participating public. Psychodrama proved to be efficient in most interventions, emphasizing its flexibility and comprehensiveness, allowing significant changes in the participants' lives, reflections on behavioral patterns and self-knowledge. The limitations found underscore the scarcity of resources, apparently financial, as a common issue in numerous articles.
\end{abstract}

KEYWORDS: Psychodrama; Sociodrama; Interventions; Limitations; Results.

\section{INTERVENCIONES DE PSICODRAMA: UNA REVISIÓN SISTEMÁTICA}

\section{RESUMEN}

El psicodrama busca conocer la realidad presentada, investigando la psique humana a través de la dramatización. Este estudio tiene como objetivo identificar intervenciones en psicodrama, sus limitaciones y resultados. Esta revisión sistemática de la literatura buscó trabajos publicados entre

\footnotetext{
1.Faculdade Meridional - Passo Fundo (RS), Brasil.

2.Universidade do Vale do Rio dos Sinos - São Leopoldo (RS), Brasil.

*Autor correspondente: vandroantonio@gmail.com

Editor de Seção: Oriana Hadler

Recebido: Dez. 07, 2020 | Aceito: Jan. 27, 2021
} 
2009 y 2019, en el Portal de Periódicos da Capes. De los 1403 estudios encontrados, 34 cumplieron los criterios de inclusión y exclusión. Tras analizar los artículos surgieron 11 categorías y se agruparon por similitud de temas o público participante. El psicodrama demostró ser eficaz en la mayoría de las intervenciones, enfatizando su flexibilidad y amplitud, permitiendo cambios significativos en la vida de los participantes, reflexiones sobre patrones de comportamiento y autoconocimiento. Las limitaciones encontradas subrayan la escasez de recursos, aparentemente económicos, como tema común en numerosos artículos.

PALABRAS CLAVE: Psicodrama; Sociodrama; Intervenciones; Limitaciones; Resultados.

\section{INTRODUÇÃO}

São consideradas psicodrama aplicações guiadas através da dramatização, nas diversas modalidades operacionais como a investigação, o aprendizado, o treinamento ou a compreensão com fins terapêuticos (Menegazzo et al., 1995). Pode ocorrer em variados campos de atuação: educacional, institucional, didático, clínico, grupal, individual, de casal e de família, dentre outros. Quando em grupo, a metodologia recomenda cinco instrumentos, que são o protagonista, a plateia ou caixa de ressonância, os egos-auxiliares, o cenário e o diretor ou coordenador. No psicodrama individual ou bipessoal, caracterizado pelo paciente e pelo terapeuta apenas, não há egos auxiliares nem plateia (Cukier, 1992).

Os instrumentos são os meios utilizados para a aplicação das técnicas psicodramáticas (Gonçalves et al., 1988). O cenário é o espaço criado conforme as necessidades terapêuticas e se constitui a partir de convenções estabelecidas entre diretor e protagonista. O protagonista é o sujeito escolhido pelo grupo para a ação dramática. Diretor é o terapeuta que coordena a sessão e tem três funções: diretor da cena, terapeuta do protagonista e do grupo e observador social. Por fim, o público ou plateia são os outros participantes da sessão, funcionando como caixa de ressonância, dividem suas emoções e percepções na última etapa da sessão, chamada de compartilhamento.

O desenvolvimento da sessão psicodramática acontece em três etapas: o aquecimento, a dramatização e o compartilhamento (Gonçalves et al., 1988). No aquecimento devem ser permitidas as manifestações de sentimentos, conflitantes ou não, que surjam entre os participantes. A dramatização, propriamente dita, pode ser entendida como tendo quatro momentos diferentes: montagem da cena, investigação, elaboração e resolução (Bustos, 2005). Na montagem da cena é avaliado o tempo em que ela ocorreu (idade do protagonista, o que acontecia naquele lugar etc.) e seu espaço cenográfico (como era o ambiente, havia portas ou janelas que merecem ser representadas por almofadas ou outro objeto?). Em seguida, na investigação, o objetivo é pesquisar e produzir a cena, com foco na concretização, quando os sentimentos que foram suscitados se concretizam a partir da dramatização, podendo-se, por exemplo, trazer à cena o protagonista no papel do sentimento, a partir da troca de papéis. $\mathrm{Na}$ elaboração, as técnicas clássicas permitem a formulação do que foi investigado na etapa anterior, almejando a reflexão. Finalmente, na resolução, podem-se buscar as realidades suplementares, que, segundo Gonçalves et at. (1988), é a dramatização do que não aconteceu na vida real. Sendo assim, é possível traduzir fatos, fantasias e lembranças que serão recriadas, com vistas a alternativas ao conflito dramático apresentado pelo protagonista nesta etapa. $\mathrm{Na}$ terceira etapa da sessão psicodramática, o compartilhamento, a solicitação a cada participante é em que ponto foi tocado, falando de si, sendo que o que menos se espera é comentar a cena dramatizada (Bustos, 2005).

É importante fazer algumas diferenciações de nomenclatura. O conceito psicodrama, pelo seu uso extensivo, sobressaiu-se e acabou representando toda a teoria criada por Jacob Levi, porém há diferenciações. O termo "Socionomia" refere-se ao "estudo das leis que regem o comportamento social e grupal" (Gonçalves et al., 1988, p. 41), que se divide em Sociodinâmica, que estuda o funcionamento ou a dinâmica das relações interpessoais, Sociometria, que busca medir essas relações através do teste sociométrico, e Sociatria, a qual está relacionada ao tratamento das relações sociais. Os autores postulam que o psicodrama busca tratar o indivíduo, tornando-o protagonista de sua história. "O psicodrama foi definido como um método de ação profunda, lidando com as relações interpessoais e as ideologias particulares, e o sociodrama como um método de ação profunda que trata das relações intergrupais e das ideologias coletivas” (Moreno, 2016, p. 411). Para Nery (2012), o 
sociodrama não tem um protagonista, mas um tema protagônico, ou seja, a dramatização das situações-problema, focado nos papéis sociais e nos sofrimentos coletivos. Vale ressaltar que existem entendimentos diversos do protagonista.

Buscar a verdade, segundo Moreno (1974), fazendo uso da dramatização, e investigar a psique humana fazendo uso da ação pode ser entendido como a finalidade da ciência do psicodrama, compreendido como um todo, no seu uso extensivo. Para Cukier (2018), o psicodrama que trata o indivíduo ou o grupo é desenvolvido a partir da aplicação da ação dramática. Segundo Rojas-Bermúdez (2016), o psicodrama não negligencia as palavras, mas utiliza-as de modo hierarquizado no contexto maior dos atos. Logo, a linguagem verbal tem seu uso entre outras linguagens, como a não verbal, e elas são empregadas na busca da verdade. A responsabilização do sujeito é pelo que é feito e dito no "aqui e agora".

Ao tratar o sujeito como resultado de interações, em seu meio, o psicodrama vê a necessidade do outro no nascer, crescer e viver (Rojas-Bermúdez, 2016). A dramatização não é do indivíduo isolado, mas em suas interações, e isso é expresso pelo grupo. Dessa forma, mesmo na terapia bipessoal (psicodramatista-cliente), o paciente/cliente irá atuar a partir de cenas da sua vida e de papéis que vivencia ou interage, evidenciando as relações daquele conflito. "O psicodrama dramatiza para desdramatizar" (Menegazzo et al., 1995, p. 80).

Para estudar o psicodrama, é imprescindível entender o conceito de "encontro", central nesta concepção, na qual se busca o resgate da espontaneidade na interação "eu-tu" (Bustos, 1979). O encontro de Moreno é uma convocação para sensibilidade com o próximo e liberta o homem espontâneo frente ao "homem-robô". Sugere nesse encontro a vivência da capacidade mútua que as pessoas têm em se colocar no lugar do outro, a empatia de mão dupla, também entendida como tele ou telessensibilidade (Gonçalves et al., 1988). O encontro autêntico que Moreno propõe é a possibilidade de relação da pessoa consigo mesma, com a humanidade e com a transcendência (Menegazzo et al., 1995). Para o encontro acontecer, devem-se ultrapassar os estereótipos, amadurecendo a liberdade, a espontaneidade e a criatividade.

Tele é a percepção empática mútua que ocorre entre duas pessoas, ou seja, em ambas as direções (Gonçalves et al., 1988). Transferência seria o enfraquecimento ou ausência dessa capacidade, ou seja, o oposto da tele. Descobrir, aprimorar e utilizar relações predominantemente télicas sobre as transferenciais é um dos objetivos do psicodrama. O encontro de Moreno é uma experiência télica. A tele pode ser assimilada como a faculdade humana de comunicação de afetos (Menegazzo et al., 1995).

Ao nascer, o bebê não dispõe de modelos para aquela situação completamente nova, e sua forma de reagir é chamada de espontaneidade (Moreno, 2016). Ela pode ser mais ou menos adequada, mas um mínimo dela é necessário já no primeiro dia de vida. A espontaneidade é a capacidade de dar respostas a situações novas, ou também novas respostas a situações antigas (Bustos, 1979). Ela pode ser treinada e aprendida através do processo psicodramático. Produzir uma ação adequada, de forma inédita, renovadora ou transformadora, é entendido como ser espontâneo. Logo, a proposta de Moreno é adequar a pessoa a ela mesma por meio da criatividade (Gonçalves et al., 1988). Para ser espontâneo, o homem precisa se relacionar de maneira mais consciente e autônoma com as conservas culturais que lhe são impostas, às quais inconscientemente se submete (Bustos, 1979).

Representar um papel no cenário psicodramático permite ao indivíduo processos cognitivos que the viabilizam a operação e reestruturação dessas respostas diante das situações (Menegazzo et al., 1995). Os autores diferenciam três instâncias, num crescente de liberdade quanto aos papéis: sua recepção, sua interpretação e sua criação. A recepção (role taking) é o aprendizado do papel por observação e imitação, sem variações do papel já pronto; a interpretação (role playing) é o jogo com os papéis apreendidos, mesclando imitação com improvisação, onde há certa liberdade; e a criação (role creating) é baseada na criatividade com novas respostas, flexibilidade emocional e comportamental. Algumas condutas de comportamento são vistas como inadequadas, patológicas, enquanto outras são estimuladas e aceitas como funcionais (Dedomênico, 2013).

Para posterior compreensão dos resultados, é imprescindível o entendimento do psicodrama mediante a delimitação deste estudo. As abordagens psicodramáticas são compreendidas como ferramentas para implementar uma filosofia de vida responsável e criativa (Blatner \& Blatner, 1996). O psicodrama e o sociodrama seguem os autores, são métodos da Sociatria e se preocupam em produzir uma terapia social que busca melhorar a autenticidade das relações, promovendo catarse coletiva. Suas intervenções têm respaldo na Sociatria e apresentam resultados terapêuticos (Gonçalves et al., 1988). Esta revisão buscou especificamente intervenções psicodramáticas, e todas elas fazem parte da clínica ampliada, que, embora aconteçam em diversos ambientes, pertencem a essa grande área psicológica. 
Dessa forma, a partir dos construtos e explanações acima abordadas, esta revisão tem como objetivos identificar, através de relatos da literatura, como são feitas as intervenções em psicodrama, buscando seus resultados e limites. Isso ocorrerá por meio da busca de literatura científica em periódicos.

\section{MÉTODO}

Para realizar esta revisão sistemática da literatura, foi utilizado o Portal de Periódicos da Capes, em vista da sua abrangência nas bases de dados nacionais e internacionais. Por meio da busca avançada, foi criada uma string a partir de palavras-chave relacionadas à delimitação proposta, com os operadores booleanos $A N D$ e $O R$, que está descrita a seguir: ("psychodrama" OR "sociodrama”) AND ("application" OR “use" OR "fields" OR "area of activity” OR "area of operation” OR "performance area” OR "practice” OR “area of practice" OR “intervention” OR “dramatic play” OR “dramatic expression”).

Foram incluídos nesta revisão bibliográfica apenas artigos de pesquisas empíricas de periódicos revisados por pares, que continham intervenções em psicodrama, escritos nos idiomas português, inglês e espanhol, publicados num período de dez anos, compreendido entre 2009 e 2019. Foram excluídos desta pesquisa artigos sem estrutura básica clara, com no mínimo Introdução, Metodologia e Resultados, bem como artigos que utilizaram, em suas intervenções, a linha teórica do psicodrama psicanalítico, por este estar baseado na psicanálise e apresentar conceitos e resultados dissonantes das outras pesquisas encontradas.

Realizou-se a busca por dois avaliadores independentes a fim de promover a seleção e avaliação mais criteriosa do material encontrado e posterior compilação dos dados. Havendo divergência de avaliações, um terceiro avaliador seria convidado a avaliar, circunstância que não foi necessária (Zoltowski et al., 2014). Foram usadas diretrizes de revisões sistemáticas para construção desta, aumentando sua capacidade de busca organizada, de forma reflexiva, crítica e compreensiva (Costa \& Zoltowski, 2014).

Conforme o fluxograma apresentado (Fig. 1), a pesquisa base obteve o resultado de 1.403 artigos. Destes, foram selecionados apenas os que possuíam em seus títulos ou abstracts as palavras "psicodrama" ou "sociodrama". A seleção seguinte se deu excluindo os estudos escritos em outros idiomas, restando 79 artigos. A partir da leitura de seus abstracts e métodos, foram selecionadas apenas pesquisas empíricas, permanecendo 58 artigos. Por fim, a partir da leitura dos mesmos, foram selecionadas intervenções, com a estrutura básica proposta e excluídas aquelas pautadas no psicodrama psicanalítico: 34 artigos atenderam aos critérios.

Para apresentação das intervenções, limitações e resultados dos artigos pesquisados, objetivos deste estudo, foram criadas 11 categorias, por similaridade de assuntos abordados ou de público participante: interfaces da regulação emocional;

\section{Fluxograma da Seleção de Artigos}

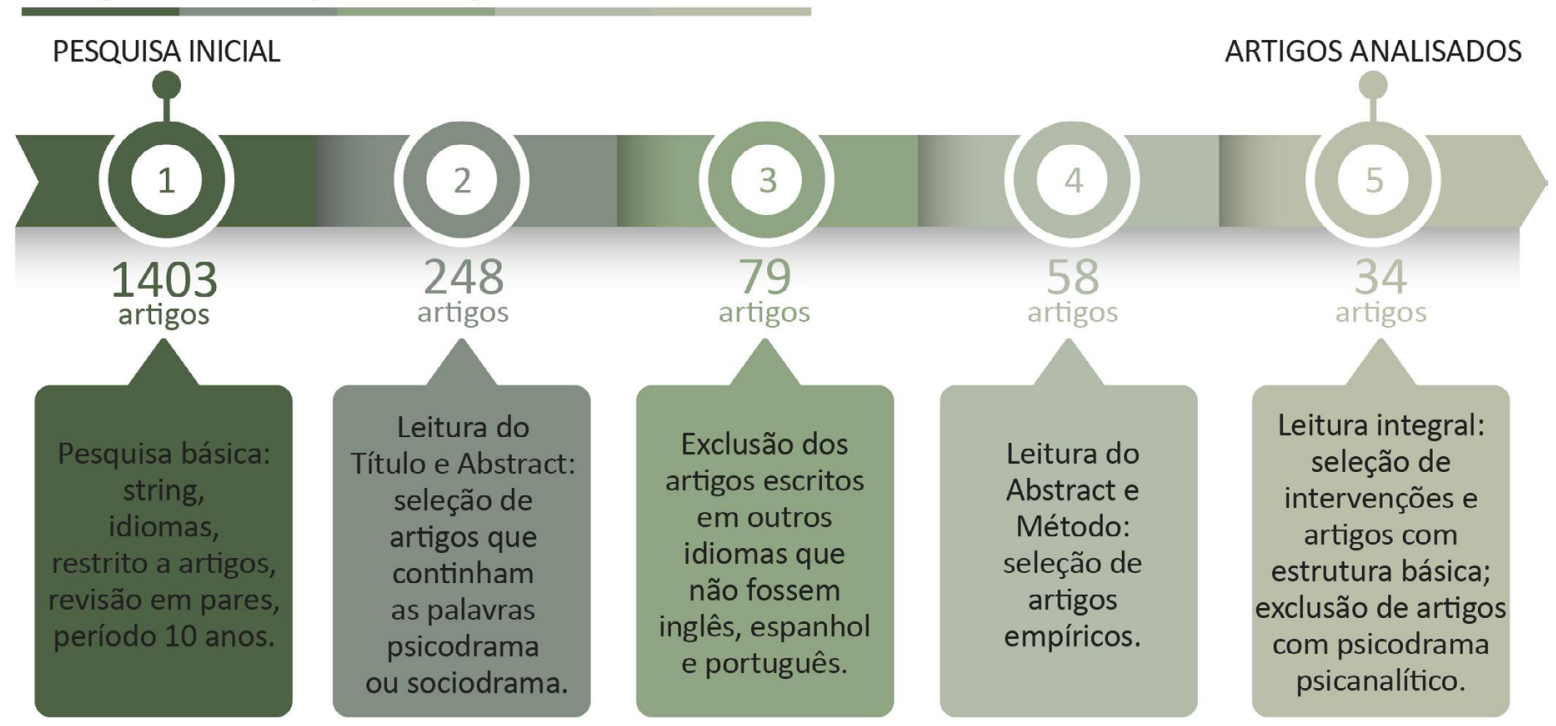

Figura 1 - Fluxograma da seleção de artigos 
aplicações na educação; contribuições no enfrentamento do estresse, ansiedade e depressão; contribuições no trabalho com crianças; contribuições no trabalho com adolescentes; enfrentamento da violência; enfrentamento à dependência; avaliação do processo terapêutico; contribuições nas organizações e no esporte; contribuição no adoecimento orgânico; e contribuição na saúde mental.

\section{RESULTADOS}

A partir da leitura das pesquisas encontradas, foi realizada uma caracterização geral dos artigos: a maior parte deles é originária da Turquia, com sete produções, seguida pela Itália e pelos Estados Unidos, com cinco artigos cada país. Do Brasil, foram encontrados apenas dois. A maioria dos artigos foi escrita na língua inglesa, somando 31 . $\mathrm{O}$ ano de maior produção foi 2018, com oito. Em relação ao delineamento metodológico, o método misto somou 14 artigos. Os instrumentos mais utilizados foram Spontaneity Assessment Inventory - Revised (SAI-R) e o Clinical Outcomes in Routine Evaluation - Outcome Measure (CORE-OM), utilizados/relatados nos mesmos quatro artigos.

$\mathrm{Da}$ análise dos artigos, emergiram as 11 categorias já mencionadas, e elas serão abordadas na sequência. Na categoria "Interfaces da regulação emocional", três estudos foram encontrados. As intervenções se preocuparam em investigar a regulação emocional em pacientes psiquiátricos (Prosen \& Jendričko, 2019), avaliar o potencial do psicodrama no sofrimento agudo neste mesmo público (Ron, 2018) e investigar o processo de resolução de dor emocional em pessoas inscritas no programa (McVea et al., 2011). As principais limitações dos estudos foram o baixo número de participantes (Prosen \& Jendričko, 2019) ou poucos casos estudados (McVea et al., 2011), além da sugestão de estudos quantitativos para melhor percepção do fenômeno (Ron, 2018). Quanto aos resultados, foi percebido que o psicodrama pode auxiliar significativamente na resolução da dor emocional, com melhorias no relacionamento interpessoal (McVea et al., 2011) e no manejo do sofrimento e isolamento agudos de pacientes psiquiátricos (Ron, 2018), bem como permitir obtenção de insights sobre os próprios pensamentos e emoções, melhorando a regulação emocional (Prosen \& Jendričko, 2019).

A categoria "Aplicações na educação" possui dois artigos. Suas intervenções se preocuparam em avaliar o impacto do programa de educação ambiental no domínio afetivo de professores (Okur-Berberoglu, 2017) e utilizar a prática sociodramática na disciplina de Expressão Dramática para desenvolver habilidades sociais e pessoais (Silva \& Leonido, 2016). Só um dos estudos apresentou limitações, que foi a inexistência de acompanhamento e os professores não terem participado da construção do programa (Okur-Berberoglu, 2017). Quanto aos resultados, foi percebido que as técnicas do sociodrama geram resultados imediatos no dia a dia dos alunos, auxiliam o crescimento pessoal e do grupo, desenvolvem o autoconhecimento e podem ser preventivas quanto a dificuldades na escola (Silva \& Leonido, 2016). Já na outra pesquisa, os principais conceitos relacionados aos domínios afetivos foram interesse, ansiedade, motivação e reclamação, e as mudanças relacionadas a eles podem ter sido mal interpretadas ou não demonstradas pelos participantes, ou é possível, ainda, que o sociodrama não tenha sido suficiente para sua identificação e avaliação (Okur-Berberoglu, 2017).

A categoria "Contribuições no enfrentamento do estresse, ansiedade e depressão" é composta por quatro artigos. As intervenções deles objetivaram avaliar o estresse, a ansiedade e a depressão através dos níveis de cortisol lingual (Erbay et al., 2018), determinar os efeitos do psicodrama em mulheres com transtorno depressivo (Ebrahimi Belil, 2011), avaliar a eficácia do psicodrama em adultos jovens ansiosos com estilos de apego pouco saudáveis (Dogan, 2010), além de explorar o sentido da vida e da morte avaliando ansiedade e alexitimia numa escola com um caso de suicídio (Testoni, Ronconi, et al., 2018). As principais limitações encontradas foram o número limitado de participantes e/ou sessões (Dogan, 2010; Erbay et al., 2018). A falta de acompanhamento em longo prazo para estabelecer a estabilidade dos resultados também foi limitante (Dogan, 2010; Testoni et al., 2018). Em relação aos resultados, os autores perceberam um declínio significativo nos níveis de estresse, ansiedade e severidade da depressão através do psicodrama (Ebrahimi Belil, 2011; Erbay et al., 2018). A melhora nos níveis de apego, de autoconhecimento, da autoconfiança, da empatia e do enfrentamento puderam ser percebidos nos adultos jovens após o programa (Dogan, 2010). Por fim, a reflexão sobre a vida e a morte diminuiu a ansiedade e contribuiu para o enfrentamento de problemas nos alunos daquela escola, através do psicodrama e de atividades artísticas (Testoni, Ronconi, et al., 2018). 
$\mathrm{Na}$ categoria "Contribuições no trabalho com crianças" há três artigos. As intervenções desses trabalhos buscaram investigar a efetividade do Play Therapy integrada ao Heart-Centered Energetic Psychodrama (HCEP) no tratamento de crianças traumatizadas (Prigel, 2017), avaliar os efeitos do psicodrama em crianças autistas (Li et al., 2015) e analisar os efeitos do psicodrama em variáveis parentais que afetam crianças com TDAH (Vural et al., 2014). As limitações foram mencionadas apenas em dois estudos: o curto período de intervenção, sem acompanhamento futuro em ambos; conhecimento limitado sobre o psicodrama interferindo na sua operacionalização ( $\mathrm{Li}$ et al., 2015) e número restrito de cuidadores interferindo na eficiência do grupo e falta de escalas para mensurar sua efetividade (Vural et al., 2014). Os principais resultados foram: através da play therapy e do psicodrama as crianças exploram, atuam e enfrentam suas dificuldades, o HCEP é um tratamento que induz a um nível profundo de cura e, quando combinados, são eficazes com crianças traumatizadas (Prigel, 2017); as barreiras sociais da criança autista diminuíram ao final do tratamento e este pode melhorar a capacidade cognitiva social (Li et al.,2015); psicopatologia e atitudes negativas são fatores parentais que têm impacto desfavorável às crianças com TDAH e o psicodrama pode ser eficaz para esses fatores (Vural et al., 2014). Outros fatores negativos podem gerar estigmatização e punição severa para essas crianças.

A categoria "Contribuições no trabalho com adolescentes" engloba três artigos desta revisão. Duas das intervenções utilizaram o instrumento Scene-Based Psychodramatic Family Therapy (SB-PFT) para melhorar o comportamento problemático de adolescentes com suas famílias (Maya, Jiménez, et al., 2018) e avaliar a variabilidade emocional de adolescentes com problemas comportamentais de acordo com eventos estressores (Maya, Lorence, et al., 2018). Outro estudo buscou avaliar a efetividade do psicodrama na resolução de conflitos em adolescentes do Ensino Médio (Karatas, 2011). As principais limitações foram a influência mútua entre participantes e a devolução de acordo com as expectativas dos pesquisadores, além da necessidade de diferentes populações de adolescentes (Maya, Jiménez, et al., 2018). Estudos longitudinais sobre eventos estressantes e sua permanência na vida dos adolescentes, além da avaliação da raiva e hostilidade, também foram uma limitação importante (Maya, Lorence, et al., 2018). Por fim, foi percebida como limitante à escala de aplicação do estudo apenas naquela instituição (Karatas, 2011). Como resultados os autores referem que pais e adolescentes perceberam mudança de atitude, melhor aceitação, comunicação familiar, expressão emocional e sentimental, além de controle de impulsos a partir do SB-PFT (Maya, Jiménez, et al., 2018). O estudo seguinte concluiu que, quando há violência familiar, os adolescentes têm mais dificuldades comportamentais e menos vínculos familiares, embora tenham melhorado suas inteligências emocionais e vínculos parentais (Maya, Lorence, et al., 2018). Por último, percebeu-se que as técnicas do psicodrama têm efeitos favoráveis nas habilidades de resolução de conflito dos adolescentes (Karatas, 2011).

A categoria "Enfrentamento da violência" é composta por cinco artigos. Suas intervenções buscaram: entender o empoderamento de mulheres abusadas através do psicodrama (Bucuţă et al., 2018); comparar os índices de espontaneidade e bem-estar psicológico em mulheres vítimas de violência doméstica (Testoni et al., 2015); analisar a incapacidade na educação de filhas de mães que sofrem violência doméstica (Guglielmin et al., 2014); avaliar a eficácia de programas de prevenção de violência em adolescentes latinos nos Estados Unidos (Smokowski \& Bacallao, 2009); e investigar os efeitos do grupo de TCC e de psicodrama nos índices de agressão em adolescentes (Karatas \& Gokcakan, 2009). As limitações ficaram restritas a dois artigos e foram apresentadas como o tamanho da amostra pequeno para generalização (Bucuţă et al., 2018) e para realizar subanálises de famílias de diferentes países de origem (Smokowski \& Bacallao, 2009). Os principais resultados foram: o psicodrama pode empoderar mulheres abusadas e estimular estratégias de enfrentamento através da confiança, da autoestima e do empoderamento (Bucuţă et al., 2018); o psicodrama parece aumentar a espontaneidade e o bem-estar psicológico, apresentando técnicas eficazes no suporte às mulheres vítimas de violência doméstica (Testoni et al., 2015); a técnica do Átomo Social pode ser útil para mulheres vítimas de violência, tornando-as conscientes das relações e dinâmicas familiares, auxiliando-as a modificar padrões transgeracionais (Guglielmin et al., 2014); o programa Entre Dos Mundos, utilizando técnicas do psicodrama, foi eficaz na diminuição do comportamento violento dos adolescentes latinos (Smokowski \& Bacallao, 2009); por fim, a abordagem cognitivo-comportamental foi mais eficaz na diminuição da agressão do que o psicodrama com os adolescentes do estudo (Karatas \& Gokcakan, 2009).

A categoria "Enfrentamento à dependência" é composta por dois artigos. Suas intervenções são: um estudo de caso em uma comunidade terapêutica para tratamento de uso de álcool e substâncias (Testoni, Cecchini, et al., 2018), e uso de 
técnicas sociodramáticas buscando reflexão para escolhas saudáveis em relação ao uso de álcool por estudantes universitários (Haleem \& Winters, 2011). As principais limitações foram o número reduzido de participantes, impedindo a generalização das intervenções (Haleem \&Winters, 2011; Testoni, Cecchini, et al., 2018) e a dificuldade na construção do tratamento pela contaminação provocada pelo dia a dia da comunidade terapêutica (Testoni, Cecchini, et al., 2018). Seus resultados demonstram que o psicodrama e o sociodrama podem ser usados nos tratamentos de dependência química com boas implicações (Haleem \&Winters, 2011; Testoni, Cecchini, et al., 2018), e suas técnicas contribuíram para os alunos refletirem sobre o uso de álcool, aumentando o comprometimento na redução de danos (Haleem \& Winters, 2011).

A categoria "Avaliação do Processo Terapêutico" é composta por três artigos. As intervenções buscaram avaliar a eficácia do psicodrama como método psicoterapêutico na solução de problemas (Gonzalez et al., 2018), examinar o engajamento psicodramático e o vínculo terapêutico entre adolescentes (Orkibi et al., 2017) e refletir sobre a mudança pessoal e o aprendizado emocional no processo terapêutico de futuros psicoterapeutas (Dima \& Bucuţă, 2012). Apenas um artigo apresentou limitações, que foi o tamanho reduzido da amostra para fins estatísticos, falta de vídeos gravados para auxílio interpretativo e estudos futuros com classificações de observadores dos comportamentos (Orkibi et al.,2017). Os resultados apresentados foram a percepção de mudanças na vida dos participantes, como maior empatia, aumento do bem-estar e espontaneidade, melhoria de objetivos terapêuticos, diminuição de sintomas e percepção do psicodrama como algo útil e com impacto transformacional na vida deles (Gonzalez et al., 2018). Foi considerado que o envolvimento dramático do cliente aumentou a probabilidade de comportamentos de exploração cognitivo-comportamentais, emocionais, insights e mudança terapêutica nas sessões, com menos resistência (Orkibi et al., 2017). Por fim, houve a percepção de que a experiência da terapia ampliou as perspectivas dos participantes sobre os problemas humanos, aumentando empatia, além do progresso no eu mais autêntico (Dima \& Bucuţă, 2012).

A categoria "Contribuições nas organizações e no esporte" é composta por quatro artigos. Suas intervenções buscaram melhorar o senso de coerência em empregados com burnout em grupo analítico e de psicodrama (Kahonen et al., 2012), a contratação de empregados para um call center (Figueira \& Figueira, 2011), a resolução de conflitos entre sócios de uma empresa (Freitas \& Guenzburger, 2009) e a melhora da relação psicossocial esportiva em times de handball e voleyball (Lupu \&Dobrescu, 2009). Apenas dois artigos apresentaram limitações: número relativamente baixo de participantes e não inclusão da análise qualitativa para explicar a quantitativa (Kahonen et al., 2012); sugerir o desenvolvimento de metodologias alternativas que incentivem o relacionamento com sócios e estudos para comparar conflitos societários entre diferentes culturas (Freitas \& Guenzburger, 2009). Seus principais resultados foram: constatação de senso de coerência maior no grupo de psicodrama que no analítico durante a intervenção e resultado inverso nos meses de acompanhamento, concluindo que os dois tipos de grupos poderiam ter efeitos prolongados quando associados (Kahonen et al., 2012); satisfação no jogo dramático como forma de escolha dos profissionais, pois permite conhecer pessoas clara, objetiva e descontraidamente (Figueira \& Figueira, 2011); conclusão de que o sociodrama é mais uma alternativa à disposição das pequenas e médias empresas para gerenciamento de conflito entre sócios (Freitas \& Guenzburger, 2009); e constatação de que o psicodrama e o sociodrama se tornaram fator de progresso, crescimento esportivo e de autoconhecimento pessoal e do grupo, além da redução de tensões, rejeições e conflitos nos times (Lupu \& Dobrescu, 2009).

A categoria "Contribuições no adoecimento orgânico" é composta por dois artigos. Nessas intervenções foi avaliada a efetividade do psicodrama no tratamento de paciente com HIV/AIDS (Karabilgin et al., 2012) e na busca do entendimento da experiência de ajustamento de pacientes com câncer a partir dos efeitos do psicodrama (Menichetti et al., 2015). Suas limitações foram: sugerir estudos adicionais nos pacientes com câncer, explorando a influência de aspectos clínicos, organizacionais e textuais no processo terapêutico (Menichetti et al., 2015), e o risco de contaminação nos resultados pelo moderador estar participando das entrevistas (Karabilgin et al., 2012). Os principais resultados desses artigos foram: a participação dos pacientes com câncer utilizando um método psicodramático gerou resultados positivos, além de apoiar a mobilização física e o senso de agir, e permitir lidar com os processos de luto (Menichetti et al., 2015); diminuição nos sintomas de depressão e no medo da morte, melhor percepção e conscientização, habilidades para lidar com as dificuldades e relacionamentos, e a melhora na qualidade de vida dos participantes sugere que o psicodrama tem um efeito positivo em pacientes com HIV/AIDS (Karabilgin et al., 2012). 
Por fim, a categoria "Contribuições na saúde mental" é composta por três artigos. Suas intervenções buscam avaliar a efetividade do psicodrama no tratamento de paciente com bulimia nervosa (Martín et al., 2017), utilização do psicodrama para diminuir a vergonha e a homofobia internalizada em homens gays (Olesen et al., 2017), e examinar a participação de assistente social no tratamento de pacientes em hospital psiquiátrico (Konopik \& Cheung, 2013). Apenas este último explicitou limitações, que foi a dificuldade de atribuir os resultados unicamente ao psicodrama pela utilização de outras modalidades de tratamento. Seus principais resultados foram: as feridas de vergonha e isolamento infringidas pela cultura sexual dominante são aliviadas pelas práticas do psicodrama (Olesen et al., 2017); grupos de psicoterapia psicodramática para tratar bulimia e transtorno da personalidade borderline podem proporcionar uma melhora clínica adicional a ambas as patologias (Martín et al., 2017); e afirmação de que o psicodrama é uma ferramenta clínica que pode gerar resultados positivos, auxiliar na abertura para o novo e no pensamento com esperança, além de evidências para o uso de técnicas psicodramáticas na melhoraria do papel do assistente social no processo terapêutico no hospital psiquiátrico (Konopik \& Cheung, 2013).

\section{DISCUSSÃO}

A utilização do psicodrama ou sociodrama foi proposta em diferentes contextos, com diferentes objetivos e finalidades, apresentando resultados promissores na maioria deles. Embora o psicodrama ainda não tenha a notoriedade de outros construtos teóricos, tais como a psicanálise ou a teoria cognitivo-comportamental, seu uso vai muito além da clínica tradicional e ele vem se consolidando como uma psicoterapia efetiva e eficaz para as mais diversas necessidades, galgando crescente visibilidade, como esta revisão pôde evidenciar.

Entre as utilizações do psicodrama há as mais comuns e esperadas, assim como existem algumas pouco conhecidas. Algumas hipóteses podem ser cogitadas para tais usos nessas duas perspectivas. Um dos usos esperados mais evidentes é a facilidade com que o psicodrama acessa processos cognitivos como as emoções durante suas dramatizações (Menegazzo et al., 1995), como nas pesquisas das categorias interfaces da regulação emocional e contribuições no trabalho com crianças. O foco dado pela teoria no aqui e agora, ou seja, o presente como momento de ação (Bustos, 1979), bem como a utilização do conceito de tele, dita como a empatia de mão dupla (Gonçalves et al., 1988), também deixa clara a opção pelo psicodrama para trabalhar com estresse, ansiedade e depressão. A constante reflexão sobre as relações entre os indivíduos e os papéis desempenhados nessas relações (Gonçalves et al., 1988) corrobora com um dos usos esperados do psicodrama, como nas categorias de enfrentamento da violência e nos trabalhos com adolescentes. Por fim, o incentivo à espontaneidade e à criatividade (Bustos, 1979; Moreno, 2016) dá respaldo para criação de novos padrões de conduta, auxiliando quem sofre com a dependência, como mostra esta categoria.

No entanto, alguns usos menos comuns também merecem destaque. Na categoria da avaliação do processo terapêutico e na contribuição nas organizações e no esporte, seu emprego ainda pode causar algum espanto, embora se ampare nos mesmos conceitos citados acima, inovando quanto à aplicação. Nesta última categoria, apesar de utilizar o psicodrama de um modo pouco esperado, apresenta novas alternativas nesses contextos, merecendo atenção. A categoria "Aplicações na educação" também traz um uso pouco esperado para o psicodrama, quando o utiliza para avaliar e melhorar o processo educativo em si, e não o desempenho de professores ou alunos. A criatividade que Moreno incentivava e provocava (Gonçalves et al., 1988) não beneficia somente os clientes e pacientes, mas a própria teoria, que se reinventa para atender a novas demandas oriundas dos contextos escolares, laborais, clínicos ou institucionais, reforçando sua terapêutica na clínica ampliada.

As limitações na pesquisa são tão esperadas quanto os resultados por elas obtidos; mesmo assim, vale algumas reflexões. Doze dos 34 artigos encontrados não explicitaram as suas limitações, o que caracteriza $35 \%$ deles. Ingenuamente poderíamos pensar que a pesquisa foi perfeita e tudo ocorreu dentro do esperado, mas não é necessária muita reflexão para perceber o engano: se esses artigos estão sem limitações, há maior probabilidade de ter havido displicência dos autores ao não mencioná-las do que de fato a sua inexistência. Conjecturar os motivos de tais lapsos seria especulação, mas vale reiterar que uma boa pesquisa deve versar sobre suas dificuldades. Nos artigos que manifestam suas limitações, as que mais se repetem são os poucos participantes no estudo, assim mencionados por eles, ou a falta de acompanhamento 
longitudinal dos resultados. Os dois casos evidenciam a dificuldade de se fazer pesquisa em vista dos custos e do apoio que ela necessita, obrigando os pesquisadores a se adaptar ao orçamento e não aos melhores resultados.

A variedade de pesquisas encontradas repercute na variedade de resultados, e serão abordados aqui os que se tornaram mais notórios. Antes, porém, deve-se ressaltar que tal variedade também evidencia a flexibilidade e empregabilidade que esta teoria apresenta, tanto em público como em contextos diversos. O psicodrama colabora de forma significativa para uma vida mais equilibrada (Bustos, 1979; Menegazzo et al.,1995), como é evidenciado na grande maioria das intervenções, trazendo menor sofrimento aos seus participantes.

Mesmo quando estão instaurados transtornos mentais, é possível beneficiar esses pacientes psiquiátricos do entendimento de ser humano (Gonçalves et al., 1988; Bustos, 2005), proposto por essa teoria, bem como por suas técnicas e práticas, não ficando o sujeito reduzido a seu sintoma, articulando-se novamente com a clínica ampliada. Ainda vale ressaltar que, quando utilizado juntamente com outras teorias, como TCC, psicanálise ou sistêmica, o psicodrama se torna um apoiador de resultados promissores, mostrando-se apto para utilização em equipes multiprofissionais do SUS, por exemplo, rompendo com lógicas tradicionais de atendimento.

\section{CONSIDERAÇÕES FINAIS}

A partir da busca por artigos com intervenções em psicodrama, foi possível encontrar uma grande variedade delas aplicadas aos mais diversos públicos, contextos e culturas. Tal evidência foi constatada ao resgatar pesquisas em 13 países com crianças traumatizadas ou autistas, adolescentes com problemas comportamentais, alunos e professores, mulheres vítimas de violência, pacientes psiquiátricos, dependentes químicos, pessoas com bulimia, com ansiedade, com AIDS, com câncer, com burnout, homens gays, ou apenas pessoas que faziam terapia nesta perspectiva teórica, dentre outros. Essa multiplicidade de pesquisas evidencia a flexibilidade e abrangência que o psicodrama vem desenvolvendo desde a sua criação. Mesmo assim, diante de tal amplitude, ainda é incontestável que outros tipos de intervenções existem e não foram recuperadas nesta revisão.

São notórias, ainda, a eficácia e a eficiência da teoria e das técnicas psicodramáticas nessa variedade de contextos. Na grande maioria dos estudos encontrados, foi constatado que o psicodrama atingiu os objetivos esperados nas pesquisas realizadas. Utilizado como único aporte teórico nas intervenções ou juntamente com outras teorias, o psicodrama permitiu mudanças expressivas na vida dos participantes e foi considerado uma alternativa promissora para a reflexão sobre padrões culturais, autoconhecimento, enfrentamento de dificuldades como estresse, ansiedade e isolamento, melhora da percepção e reconhecimento emocional, além de crescimento pessoal como um todo.

Em relação às limitações apresentadas nos artigos, há uma ponderação manifesta. De acordo com os principais fatores limitantes, fica claro que fazer pesquisa, em qualquer lugar do mundo, enfrenta no geral um problema em comum: escassez de recursos, aparentemente financeiros. As limitações que mais se repetiram nos artigos refletem essa constatação. A ciência no Brasil enfrenta inúmeras adversidades que se acentuam nos dias atuais, criando nos pesquisadores a sensação de que somente aqui tais dificuldades existem; no entanto, mesmo em países desenvolvidos há similaridades nesse ponto.

As limitações deste estudo, no entanto, afastam-se um pouco das encontradas nos artigos pesquisados. A primeira é a restrição do escopo de busca, que por ter utilizado apenas o Portal da Capes, pode ter desconsiderado intervenções que enriqueceriam esta revisão, bem como seu debate. Na etapa de exclusão de outros idiomas, identificou-se um grande número de produções no idioma alemão, e essa pode ser a segunda limitação, que, se fosse suprida, acrescentaria riqueza nos resultados. Por fim, sugere-se maior divulgação e publicação de intervenções baseadas em psicodrama, visto que poucas foram encontradas e seu impacto é de grande relevância, merecendo assim a devida disseminação no mundo acadêmico.

\section{CONTRIBUIÇÃO DOS AUTORES}

Conceitualização, Fernandes VA, Cenci CMB, Gaspodini IB; Investigação, Fernandes VA, Cenci CMB, Gaspodini IB; Escrita - Rascunho Original, Fernandes VA, Cenci CMB, Gaspodini IB; Escrita - Revisão e Edição, Fernandes VA, Cenci CMB, Gaspodini IB. 


\section{AGRADECIMENTOS}

Merece o agradecimento todos que contribuíram direta ou indiretamente para esta pesquisa, em especial o Psicólogo e Psicodramatista Rodrigo Iran Melara Simões, pela sua significativa colaboração na construção teórica deste projeto.

\section{DISPONIBILIDADE DE DADOS DE PESQUISA}

Não se aplica.

\section{FINANCIAMENTO}

Não se aplica.

\section{REFERÊNCIAS}

Blatner, A., \& Blatner, A. (1996). Uma visão global do psicodrama - Fundamentos históricos, teóricos e práticos. (P. S. Dantas Jr., Trad.). Ágora.

Bucuţă, M. D., Dima, G., \& Testoni, I. (2018). “When You Thought That There Is No One and Nothing”: The Value of Psychodrama in Working With Abused Women. Frontiers in Psychology, 9(1518), 1-16. https://doi.org/10.3389/ fpsyg.2018.01518

Bustos, D. M. (1979). Psicoterapia psicodramática. (M. P. Navarro, Trad.). Brasiliense.

Bustos, D. M. (2005). O psicodrama: Aplicaçôes da técnica psicodramática (3ª ed.). (L. Neves \& R. Rejtman, Trad.). Ágora.

Costa, A. B., Zoltowski, A. P. C. (2014). Como escrever um artigo de revisão sistemática. In S. H. Koller, M. C. P. de P. Couto, J. V. Hohendorff (Orgs.), Manual de produção científica. Penso.

Cukier, R. (1992). Psicodrama bipessoal: Sua técnica, seu terapeuta e seu paciente. Ágora.

Cukier, R. (2018). Vida e clínica de um psicoterapeuta. Ágora.

Dedomênico, A. M. (2013). A funcionalidade do conceito de papel. Revista Brasileira de Psicodrama, 21(2), 81-92. https://bit.ly/2T45V9k

Dima, G., \& Bucuţă, M. D. (2012). The experience of therapeutic change for psychologists preparing to become psychotherapists. Procedia: Social and Behavioral Sciences, 33, 672-676. https://doi.org/10.1016/j.sbspro.2012.01.206

Dogan, T. (2010). The effects of psychodrama on young adults' attachment styles. The Arts in Psychotherapy, 37(2), 112119. https://doi.org/10.1016/j.aip.2010.02.001

Ebrahimi Belil, F. (2011). The effects of psychodrama on depression among women with chronic mental disorder. European Psychiatry, 26, 621. https://doi.org/10.1016/S0924-9338(11)72327-6

Erbay, L. G., Reyhani, İ., Ünal, S., Özcan, C., Özgöçer, T., Uçar, C., \& Yıldız, S. (2018). Does Psychodrama Affect Perceived Stress, Anxiety-Depression Scores and Saliva Cortisol in Patients with Depression? Psychiatry investigation, 15(10), 970-975. https://doi.org/10.30773/pi.2018.08.11.2

Figueira, A. C. de A., \& Figueira, T. de A. (2011). A Seleção Profissional amenizando Conflitos no Ambiente de Trabalho: Contribuições do Psicodrama. Interpersona: An International Journal on Personal Relationships, 5(1), 53-67. https://doi.org/10.5964/ijpr.v5i1.58

Freitas, J. A. de S. B., \& Guenzburger, H. L. R. (2009). O não reconhecido conflito societário: Uma intervenção sociodramática. Revista de Gestão USP, 16(3), 31-47. https://doi.org/10.5700/rege371

Gonçalves, C. S., Wolff,J. R., \& Almeida,W. C. da (1988). Lições de psicodrama: Introdução ao pensamento de J. L. Moreno ( $2^{\mathrm{a}}$ ed.). Ágora. 
Gonzalez, A-J., Martins, P., \& De Lima, M. de P. (2018). Studying the efficacy of Psychodrama with the hermeneutic single case efficacy design: results from a longitudinal study. Frontiers in Psychology, 9(1662). https://doi.org/10.3389/ fpsyg.2018.01662

Guglielmin, M. S., Testoni, I., Pogliani, I., Prandelli, M., Bertoldo, A., \& Verdi, S. (2014). The mother who cannot provide liberation: Family atom analysis of women victims of domestic violence. La Camera Blu: Rivista di Studi di Genere, 10(10). https://doi.org/10.6092/1827-9198/2566

Haleem, D. M., \& Winters, J. (2011). A Sociodrama: An Innovative Program Engaging College Students to Learn and Self-Reflect About Alcohol Use. Journal of Child and Adolescent Psychiatric Nursing, 24(3), 153-160. https://doi. org/10.1111/j.1744-6171.2011.00289.x

Kahonen, K., Naatanen, P., Tolvanen, A. \& Salmela-Aro, K. (2012). Development of sense of coherence during two group interventions. Scandinavian Journal of Psychology, 53, 523-527. https://doi.org/10.1111/sjop.12020

Karabilgin, Ö. S., Gökengin, G. B., Doğaner, İ., \& Gökengin, D. (2012). The effect of psychodrama on people living with HIV/AIDS. European Journal of Psychotherapy E Counselling, 14(4), 317-333. https://doi.org/10.1080/1364253 7.2012.734529

Karatas, Z. (2011). Investigating the effects of group practice performed using Psychodrama techniques on adolescents' conflict resolution skills. Educational Sciences: Theory and Practice, 11(2), 609-614. https://bit.ly/2FKcz1q

Karatas, Z., \& Gokcakan, Z. (2009). A comparative investigation of the effects of Cognitive-Behavioral group practices and psychodrama on adolescent aggression. Educational Sciences: Theory and Practice, 9(3), 1441-1452. https:// bit.ly/31kvEin

Konopik, D. A., \& Cheung, M. (2013). Psychodrama as a social work modality. Social Work, 58(1), 9-20. https://doi. org/10.1093/sw/sws054

Li, J., Wang, D., Guo, Z., \& Li, K. (2015). Using psychodrama to relieve social barriers in an autistic child: A case study and literature review. International Journal of Nursing Sciences, 2(4), 402-407. https://doi.org/10.1016/j. ijnss.2015.08.008

Lupu, G., \& Dobrescu, T. (2009). Applying the sociodramas and psychodramas with the purpose of improving the psychosocial relations in sports teams. Ovidius University Annals, Series Physical Education and Sport/Science, Movement and Health, 9(2), 281-285. https://bit.ly/31kvozV

Martín, I. B., Gómez, E. G., Itúbide, K. E., Conde, B. U., Besteiro, P. B., Franco, C. P., \& Jorge, P. G. (2017). An expressive group approach to borderline personality disorder in patients with bulimia nervosa: A clinical case. European Psychiatry, 41, S547.https://doi.org/10.1016/j.eurpsy.2017.01.768

Maya, J., Jiménez, L., Lorence, B., Del Moral, G., \& Hidalgo, V. (2018). Scene-based psychodramatic family therapy with troubled adolescents and parents: A pilot study. Family Process, 10(10),1-16. https://doi.org/ 10.1111/famp.12401

Maya, J., Lorence, B., Hidalgo, V., \& Jiménez, L. (2018). The role of psychosocial stress on a family-based treatment for adolescents with problematic behaviors. International Journal of Environmental Research and Public Health, 15(1867). https://doi.org/10.3390/ijerph15091867

McVea, C. S., Gow, K., \& Lowe, R. (2011). Corrective interpersonal experience in psychodrama group therapy: A comprehensive process analysis of significant therapeutic events. Psychotherapy Research, 21(4), 416-429. https://doi.or $\mathrm{g} / 10.1080 / 10503307.2011 .577823$

Menegazzo, C. M., Tomasini, M. A., \& Zuretti, M. M. (1995). Dicionário de psicodrama e sociodrama. (M. Lopes, M. Carbajal, V. Caputo, Trad.). Ágora.

Menichetti, J., Giusti, L., Fossati, I., \& Vegni, E. (2015). Adjustment to cancer: exploring patients' experiences of participating in a psychodramatic group intervention. European Journal of Cancer Care, 25(5), 903-915. https://doi. org/10.1111/ecc.12412

Moreno, J. L. (1974). Psicoterapia de Grupo e Psicodrama. Mestre Jou.

Moreno, J. L. (2016). Psicodrama (14a ed.). (A. Cabral, Trad.). Cultrix. 
Nery, M. da P. (2012). Sociodrama. In M. da P. Nery, M. I. G. Conceição (Orgs.), Intervenções grupais: O psicodrama e seus métodos. Ágora.

Okur-Berberoglu, E. (2017). Outdoor experiential environmental education: An adult-centred intervention for the affective domain. International Electronic Journal of Environmental Education, 7(1), 34-58. https://bit.ly/3jdlA0H

Olesen, J., Campbell, J., \& Gross, M. (2017). Using action methods to counter social isolation and shame among gay men. Journal of Gay \& Lesbian Social Services, 29(2). https://doi.org/10.1080/10538720.2017.1294518

Orkibi, H., Azoulay, B., Regev, D., \& Snir, S. (2017). Adolescents' dramatic engagement predicts their in-session productive behaviors: A psychodrama change process study. The Arts in Psychotherapy, 55. https://doi.org/10.1016/j. aip.2017.04.001

Prigel, M. (2017). Integrating play therapy and heart-centered energetic psychodrama: A profound treatment for traumatized children. Journal of Heart Centered Therapie, 20(2). https://bit.ly/3kcce6D

Prosen, S., \& Jendričko, T. (2019). A Pilot Study of Emotion Regulation Strategies in a Psychodrama Group of Psychiatric Patients. Journal of Creativity in Mental Health, 14(1), 2-9. https://doi.org/10.1080/15401383.2018.1486 260

Rojas-Bermúdez, J. (2016). Introdução ao psicodrama. (J. M. D’Alessandro, Trad.). Ágora.

Ron, Y. (2018). Psychodrama's Role in Alleviating Acute Distress: A Case Study of an Open Therapy Group in a Psychiatric Inpatient Ward. Frontiers in Psychology, 9(2075). https://doi.org/10.3389/fpsyg.2018.02075

Silva, D. F., \& Leonido, L. (2016). A prática do sociodrama em contexto escolar. European Review of Artistic Studies, 7(2), 23-50. https://doi.org/10.37334/eras.v7i2.153

Smokowski, P. R., \& Bacallao, M. (2009). Entre Dos Mundos/Between Two Worlds Youth Violence Prevention: Comparing Psychodramatic and Support Group Delivery Formats. Small Group Research, 40(1), 3-27. https://doi. org/10.1177/1046496408326771

Testoni,I., Armenti, A., Ronconi, L., Verdi, S., Wieser, M., Bucuta,M., Moita, G., \& Tarashoeva, G. (2015). Developing spontaneity and well-being in women victims of domestic violence. La Camera Blu. Rivista Di Studi Di Genere, 10(11). https://doi.org/10.6092/1827-9198/2824

Testoni, I., Cecchini, C., Zulian, M., Guglielmin, M. S., Ronconi, L., Kirk, K., Berto, F., Guardigli, C., \& Cruz, A. S. (2018). Psychodrama in therapeutic communities for drug addiction: A study of four cases investigated using idiographic change process analysis. The Arts in Psychotherapy, 61, 10-20. https://doi.org/10.1016/j.aip.2017.12.007

Testoni, I., Ronconi, L., Palazzo, L., Galgani, M., Stizzi, A., \& Kirk, K. (2018). Psychodrama and Moviemaking in a Death Education Course to Work Through a Case of Suicide Among High School Students in Italy. Frontiers in Psychology, 9(441). https://doi.org/10.3389/fpsyg.2018.00441

Vural, P., Akkaya, C., Küçükparlak, I., Ercan, I., \& Eracar, N. (2014). Psychodramatic group psychotherapy as a parental intervention in attention deficit hyperactivity disorder: A preliminary study. The Arts in Psychotherapy, 41(3), 233-239. https://doi.org/10.1016/j.aip.2014.02.004

Zoltowski, A. P. C., Costa, A. B., Teixeira, M. A. P., \& Koller, S. H. (2014). Qualidade metodológica das revisões sistemáticas em periódicos de psicologia brasileiros. Psicologia: Teoria e Pesquisa, 30(1), 97-104. https://doi.org/10.1590/ S0102-37722014000100012 